\title{
The Effects of Maturation on the Colonic Microflora in Infancy and Childhood
}

\author{
P. Enck, ${ }^{1}$ K. Zimmermann, ${ }^{2}$ K. Rusch, ${ }^{2}$ A. Schwiertz, ${ }^{2}$ S. Klosterhalfen, ${ }^{1,3}$ and J. S. Frick ${ }^{4}$ \\ ${ }^{1}$ Department of Internal Medicine VI, University Hospital Tübingen, Frondsbergstrasse 23, 72076 Tübingen, Germany \\ ${ }^{2}$ Symbio Herborn Group GmbH, 35745 Herborn, Germany \\ ${ }^{3}$ Institute of Clinical Neurology and Medical Psychology, University of Düsseldorf, 40225 Düsseldorf, Germany \\ ${ }^{4}$ Institute for Medical Microbiology and Hygiene, University Hospital Tübingen, 72076 Tübingen, Germany \\ Correspondence should be addressed to P. Enck, paul.enck@uni-tuebingen.de
}

Received 21 April 2009; Revised 1 July 2009; Accepted 6 July 2009

Recommended by Ronnie Fass

\begin{abstract}
The composition of colonic mircoflora and its changes with maturation have rarely been investigated in large samples. Methods. We used conventional microbiological testing to analyse the colonic flora (Kyberstatus, Institut forMicroecology, Herborn, Germany) of stool samples from 12484 children with different intestinal and nonintestinal diagnoses. Stool samples were analysed for total colony forming units (CFU) (per g stool) and the abundance of Bifidobacteria, Bacteroides sp., Escherichia coli, Enterococcus sp., and Lactobacillus sp. with respect to age, gender. A subset of 1089 infants was analysed for monthly changes within the first year of life. Results. Total CFU and individual microbial species were highest during the first year of life, decreased within the first 2 years, and then stabilized for the remaining childhood. In infants, the total CFU rose until month 5 , declined with weaning, and peaked at 9-10 months. Significant effects of age, but not of gender, were found in Bacteroides sp. and Lactobacilli. However Bacterioids sp. and Lactobacilli increased with age, while Enterococci and E. coli decreased, and Bifidobacteria remained stable. Conclusion. Colonic microflora show both a bacteria-specific and general pattern of maturation which is most profound within the first year.
\end{abstract}

Copyright (C) 2009 P. Enck et al. This is an open access article distributed under the Creative Commons Attribution License, which permits unrestricted use, distribution, and reproduction in any medium, provided the original work is properly cited.

\section{Introduction}

Age-related changes in the abundance of nonpathogenic bacteria found in the human colon have rarely been investigated in larger samples, neither in patients with intestinal and nonintestinal disorders nor in healthy subjects. In contrast, the effects of maturation on colonic flora in infants and children have occasionally been studied, but mostly in small cohorts of children.

Deviations in colonic flora have been shown to be responsible for respiratory diseases [1], intestinal diseases [2], and, especially, dermatologic disorders [3-6] in the pediatric population. It has, for example, been argued that the amount of exposure to commensal and pathogenic bacteria in the very early days of life, such as following a Cesarian section, [7] may contribute to the occurrence of atopic dermatitis later in life [8]. A similar argument is the basis for the "hygiene hypothesis" [9], which argues that greater exposure to environmental pathogens during childhood, for example, while growing up on a farm or having contact with animals, would protect for immunologically mediated diseases later in life $[10,11]$, but this is still a matter of debate [12]. However, the importance of commensal microbiota for the development of normal innate immunity is well established [13].

One reason for the relative absence of large-scale investigations is the suspicion that bacterial colonies found in stool samples would not remain stable during transport and storage; therefore. providing an inaccurate estimate of bacterial counts when conventional microbiological tools are used. Hence, commercial assays for fecal microbiota have not been a widely accepted diagnostic tool in routine clinical assessment. One exception is the use of fecal microbiota in the diagnosis of the inflammatory bowel diseases, especially Crohn's disease and ulcerative colitis, since these are strongly associated with Clostridium diff. colonization in both children [14] and adults [15].

Both the recent interest in prebiotic and probiotic treatment for functional intestinal $[16,17]$, other intestinal 
[18-21], and nonintestinal disorders [12-26], along with the availability of new molecular biological tools that are able to count different species, identify genetically different subspecies within each strain, and characterize normal human colonic microbiota and its variability, have created a new surge in research. Investigations regarding the effect of maturation on microbiota during childhood [27] and its change with aging [28] have recently been performed. However, as with most studies based on conventional microbiology, sample sizes have remained rather small thus far, as PCR technology is not yet easily available, and microarray chip technology that allows for the assessment of all bacterial species known to inhabit the human colon (in the range of $>1000$ ) is still very costly. This may, however, change in the near future.

The few studies that have assessed the effects of maturation on human fecal microbiota have shown that some bacterial species decline in their abundance with age, while other do not. This paper presents data from a large $(>12,000$ samples) conventional microbiological database of children with various intestinal and nonintestinal symptoms and diagnoses. Respective data regarding adult populations have recently been published [29]. The underlying hypothesis of this analysis is that the average bacterial abundance found in patients with various diseases and medical conditions may represent an approximation of what may be called the "normal" human fecal microbiota. Our assessment is based on conventional microbiological analyses that were performed in a commercial laboratory with GLP certification during the course of one year.

\section{Material and Methods}

2.1. Collection of Stool Samples for Microbiological Analysis. During the course of one year (2006), all fecal samples that were submitted by general practitioners for routine industrial microbiological analysis of nonpathogen colonic bacterial flora (Kyberstatus, Institute for Microecology, Herborn, Germany) were included in the study. In general, samples reached the laboratory within one day and were processed immediately.

To ensure that the transport did not have any effect on the cultured species, a storage study was performed with 20 fresh samples. In short, $0.2 \mathrm{~g}$ of faeces was serially diluted in $1 \mathrm{~mL}$ of phosphate-buffered saline (PBS, $\mathrm{pH}$ 7.2). The solution was vortexed for 5 seconds and serially diluted (to $10^{-9}$ ) in PBS, $\mathrm{pH}$ 7.2. One $\mathrm{mL}$ of each dilution was plated onto enrichment or selective agar media.

The remaining feces were stored for three days at a temperature of $25^{\circ} \mathrm{C}$, which represents the average temperature during shipment. Following the incubation period, the samples were processed as described and the results were compared. No significant discrepancy in the cell counts of the investigated microbiota could be detected within two days. Thus, it was concluded that a shipment of less than two days will have no effect on the composition of the cultivable microbiota. Only samples which arrived within one or two days of shipment were included in the study.
2.2. Identification and Enumeration of Microorganisms. Viable bacterial cell counts in feces were enumerated on the following selective media: Columbia blood agar (total cell count; BioMerieux, Nürtingen, Germany), U3G agar (enterobacteriacae, enterococci; Heipha, Heidelberg, Germany), Rogosa agar, (lactobacilli; Heipha), DIC agar (bifidobacteria; Heipha), Schaedler agar (bacteroides; Heipha), and SPM agar (clostridia; Heipha). Fecal samples were serially diluted in $1 \mathrm{~mL}$ of phosphate-buffered saline (PBS, $\mathrm{pH}$ 7.2) and subsequently plated on selective agar plates by a fully automated spiral plater capable of plating 12 agar plates simultaneously. Subsequently, the plates were incubated under either aerobic or anoxic conditions at $37^{\circ} \mathrm{C}$ for at least two days. Bacteria were first identified by Gram staining and colony morphologies. Additionally, identifications were performed by the API and VITEK systems (bioMérieux). All counts were recorded as the numbers of $\log _{10} \mathrm{CFU}$ per $\mathrm{mL}$ of sample.

The following bacteria were routinely analyzed: Clostridium sp., Bifidobacteria, Bacteroides sp., subdominant (E. coli, Enterococcus sp., Lactobacillus sp.), and other bacteria (Pseudomonas sp., Klebsiella sp., Proteus sp., Citrobacter sp., aerobic bacteria). Only bacteria that were identified in at least $50 \%$ of the respective subsamples (mentioned hereafter) were included into further analysis.

2.3. Additional Data. Since samples were from patients with various clinical diseases (Table 1), we collected additional data that was reported by the referring physician. This included age, gender, the presumed clinical diagnosis, and stool consistency and frequency. Stool pH was determined in the laboratory.

2.4. Data Analysis. After the data had been made anonymous, it was provided for further statistical analysis. Prior to the analysis, the sample was screened for identical patient IDs, and any second or subsequent analysis was excluded. Incomplete datasets were also excluded, except those where only gender information was missing.

The total age distribution allowed for the identification of a childhood sample $(n=12.484)$ (Figure 1$)$ and an adult sample $(n=35.292)$. The adult study has been published recently [29]. A subset of 1089 infants (<1 year) was identified for the analysis of monthly changes within the first year of life.

Total CFU was analysed as well as the bacterial abundance for the following microbiota; Bifidobacteria, Bacteroides sp., lactobacilli, E. coli, and Enterococcus sp. The percentage of subjects in which Clostridium sp. was identified was evaluated as well.

Prior to statistical analysis, data were normalized. The CFU of individual cell populations were compared to the total CFU identified by calculating the relative "abundance" of each bacterial species as ((specific CFU/total CFU)*100).

The subsets (infants, children) were analyzed separately by ANOVAs for each bacterial count (abundance) with the between factors "age" (in monthly intervals for the infants, in yearly intervals for children) and "gender." Post-hoc $t$ 
TABLE 1: Major diagnoses in children that initiated the stool analysis.

\begin{tabular}{|c|c|c|}
\hline Organ classes & Main diagnoses & Children \\
\hline \multirow{8}{*}{ Gastrointestinal } & Unspecified & 1862 \\
\hline & Irritable Bowel Syndrome & 1188 \\
\hline & Crohn's disease & 27 \\
\hline & Ulcerative colitis & 64 \\
\hline & Diarrhea & 771 \\
\hline & Constipation & 296 \\
\hline & Candida & 1061 \\
\hline & Food Intolerance & 238 \\
\hline \multirow{4}{*}{ Respiratory } & Unspecified & 663 \\
\hline & Bronchitis & 294 \\
\hline & Sinusitis & - \\
\hline & Asthma & 103 \\
\hline \multirow{3}{*}{ Urogenital } & Unspecified & 27 \\
\hline & Cystitis & - \\
\hline & Genital mycosis & - \\
\hline \multirow{4}{*}{ Dermatologic } & Unspecified & 40 \\
\hline & Dermatitis & 256 \\
\hline & Psoriasis & 28 \\
\hline & Acne & - \\
\hline \multirow{7}{*}{ Allergic } & Allergy, unspecified & 229 \\
\hline & Neurodermititis & 2184 \\
\hline & Recurrent urticaria & - \\
\hline & Pollinosis & - \\
\hline & Allergic asthma & 230 \\
\hline & Food allergies & 209 \\
\hline & Respiratory allergies & 106 \\
\hline Rheumatologic & Arthritis & - \\
\hline \multirow{3}{*}{ Others } & Infection defence weakness & 402 \\
\hline & Autoimmune diseases & - \\
\hline & Malignom & - \\
\hline Missing & & 421 \\
\hline \multirow{2}{*}{ Total } & & 10699 \\
\hline & & $(85 \%)^{*}$ \\
\hline
\end{tabular}

* The remaining to the total of 12.484 are cases with unknown diagnoses.

tests with Bonferroni correction to account for multiple comparisons were used to test for single differences (between months and years, resp.) within each dataset. Pearson's $r$ was computed to test for intercorrelation between single measures. All data are given as mean \pm SEM. A threshold of 0.05 was set to indicate statistical significance in all tests. All data were analyzed using the SPSS Version 13 Statistical Package.

\section{Results}

From the sample of $47,775, n=12483$ were children and adolescents less than 18 years, and among them, $n=1089$ were infants and babies less than one year of age. Their stool samples were analyzed for a variety of clinical conditions (see

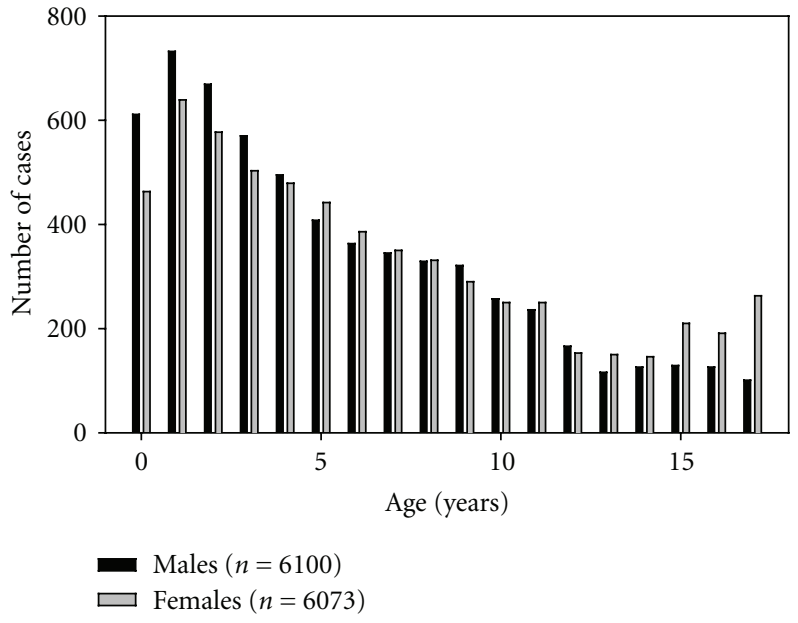

Figure 1: Age distribution (in years) in male and female subjects between 1 and 18 years.

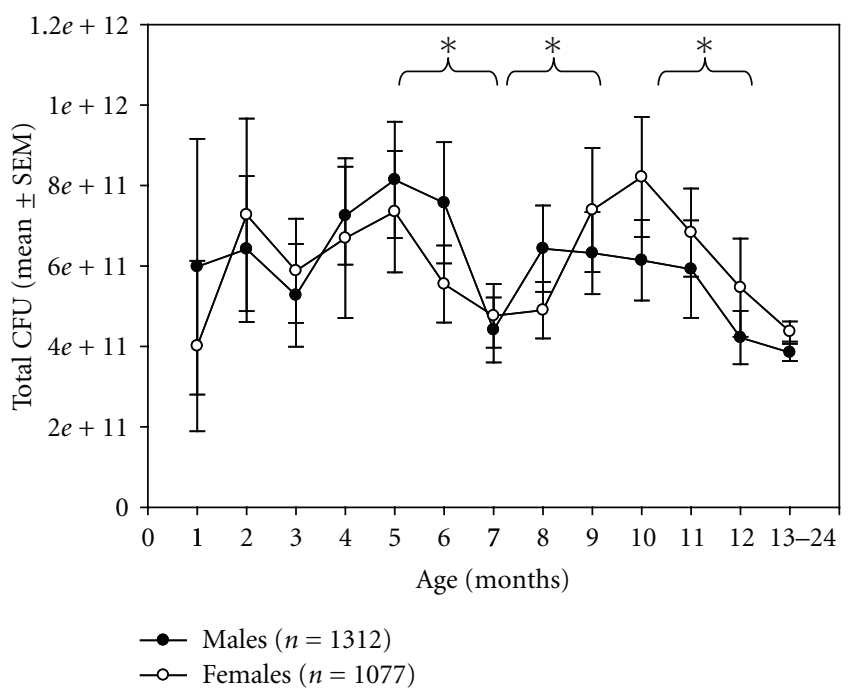

Figure 2: Total CFU (mean \pm SEM) by age (months) and by gender. " $*$ " indicate significant difference in post-hoc $T$-test (Bonferroni corrected). A number of boys and girls are infants (604: $459 \mathrm{~m}: \mathrm{f})$ plus children up to 2 years.

Table 1), but without any documentation of the diagnostic accuracy.

3.1. Infants. The infant group ( $\leq 1$ year) included 604 males and 459 females, $6.47 \pm 0.92$ months of age. ANOVA revealed a significant effect of age $(F=3.94, P<001)$, but not of gender on total CFU (Figure 2). As can be seen, CFU increased within the first few months, declined to a first low at the time of weaning, and exhibited a second peak at months 10 and 11 . It steadily declined thereafter to reach stable levels at age 5 or 6 (described in what follows).

ANOVA showed significant effects of age, but not of gender on individual bacterial abundance. All bacteria except Lactobacilli were present within the first month of life. However, Enterococci and E. coli decreased $(F=3.098, P<$ 


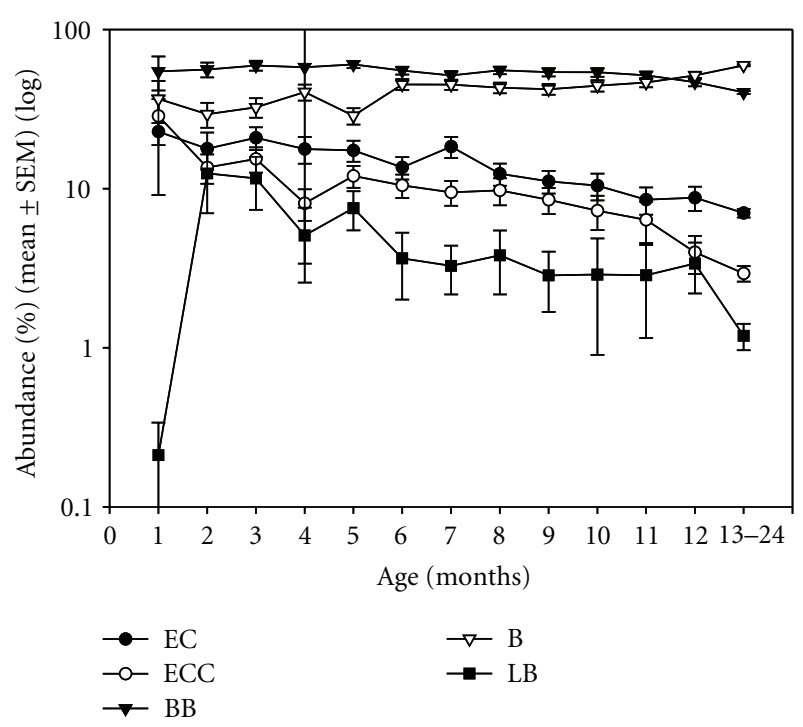

Figure 3: Abundance (\%) (mean \pm SEM) by age (months) of $E$. coli, Enterococcus sp., as well as Lactobacillus sp., Bifidobacteria, and Bacteroides sp.

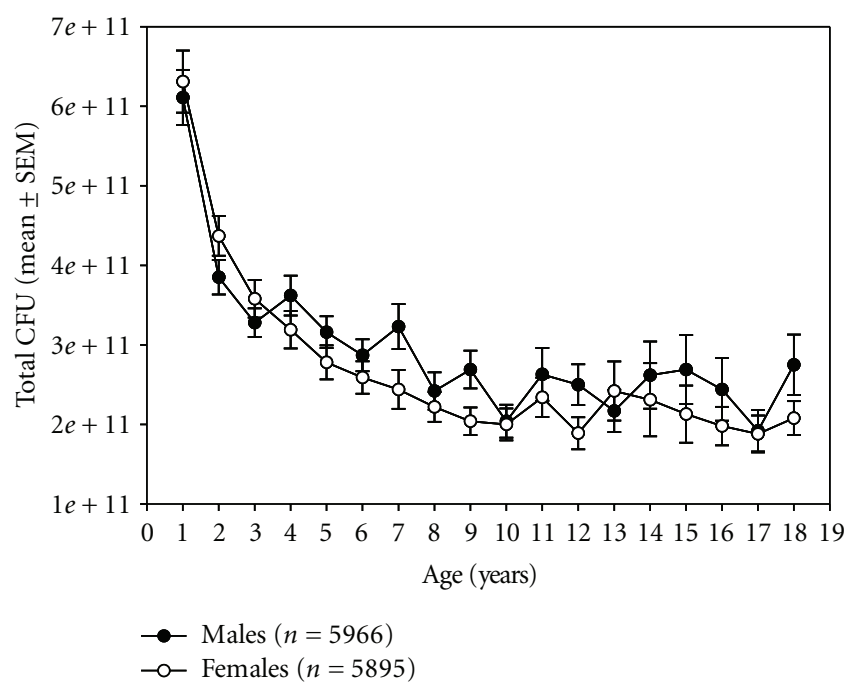

FIgURE 4: Total CFU (mean \pm SEM) by age (years) and by gender.

.001 and $F=2.884, P=.001$, resp.) while Bacteroides sp. increased $(F=3.14, P<.001)$, and Bifidobacteria remained stable $(F=1.226, P=.265)$ within the first year. Lactobacilli existed at high concentrations at month 2, but steadily decreased thereafter $(F=2.143, P=.016)$ (Figure 3). The percentage of Clostridium sp. declined from 3 to $2 \%$.

3.2. Children. For all the 12,483 children $(6.02 \pm 0.04$ years, $6100: 6074$ male: female, remaining $=$ missing $)$, total CFU significantly declined $(F=126.74, P<.001)$ from year 1 to year 10 and remained stable thereafter. Overall, females had a significantly lower bacterial count than males $(F=19.24$, $P<.001$ ) (Figure 4).

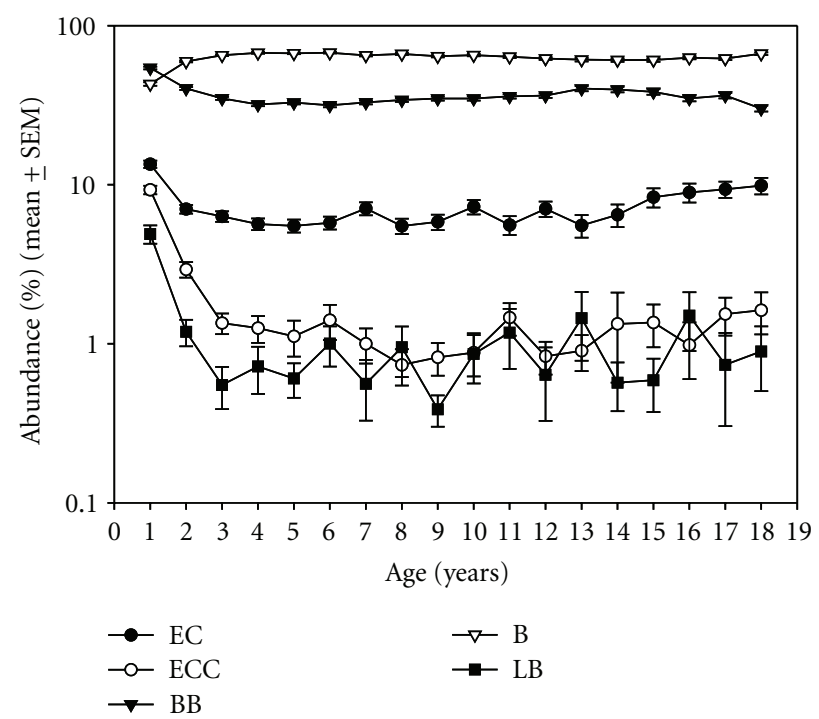

Figure 5: Abundance (\%) (mean \pm SEM) by age (years) of $E$. coli, Enterococcus sp., as well as Lactobacillus sp., Bifidobacteria, and Bacteroides sp.

Individual bacteria abundance showed the same agerelated decline within the first few years for Enterococci $(F=37.188, P<.001)$ and Lactobacilli $(F=9.888$, $P<.001)$, while E. coli moderately decreased $(F=8.679$, $P<.001)$. Given the high proportion of their specific $\mathrm{CFU}$, the time profile of Bifidobacteria and Bacteroides sp. was almost complementary. Bifidobacteria decreased overall $(F=34.051, P<.001)$, while Bacteroides sp. increased their proportion $(F=43.465, P<.001)$ (Figure 5). An independent effect of gender was noted for Bifidobacteria $(F=4.982, P=.026)$ and $E$. coli $(F=12.203, P=.001)$ with higher values for Bifidobacteria, but lower values for $E$. coli in males (data not shown). The percentage of Clostridium $s p$. remained low at around $2 \%$ (data not shown).

Stool consistency (rated between $1=$ solid and $5=$ liquid) decreased $(F=16.108, P<.001)$ and was significantly lower in females $(F=51.735, P<.001)$. Stool $\mathrm{pH}$ increased within the first years $(F=74.425, P<.001)$ and was overall higher in females $(F=23.998, P<.001)$ (Figures 6(a) and 6(b)).

Moderate negative correlations were found between $\mathrm{pH}$ and Bifidobacteria $(r=-.33, P<.001)$, and positive correlations between $\mathrm{pH}$ and $E$. coli $(r=.24, P<.001)$.

\section{Discussion}

Newborns are germfree at the time birth, and acquire bacteria from their immediate environment within the first hours of life: from their mothers' vaginal and fecal flora, and from the hospital or home environment, depending on the mode and location of delivery. Other factors that can influence the composition of the intestinal microflora in newborns are the environment during birth, maturity, hygiene measures, and the type of infant feeding [30]. 


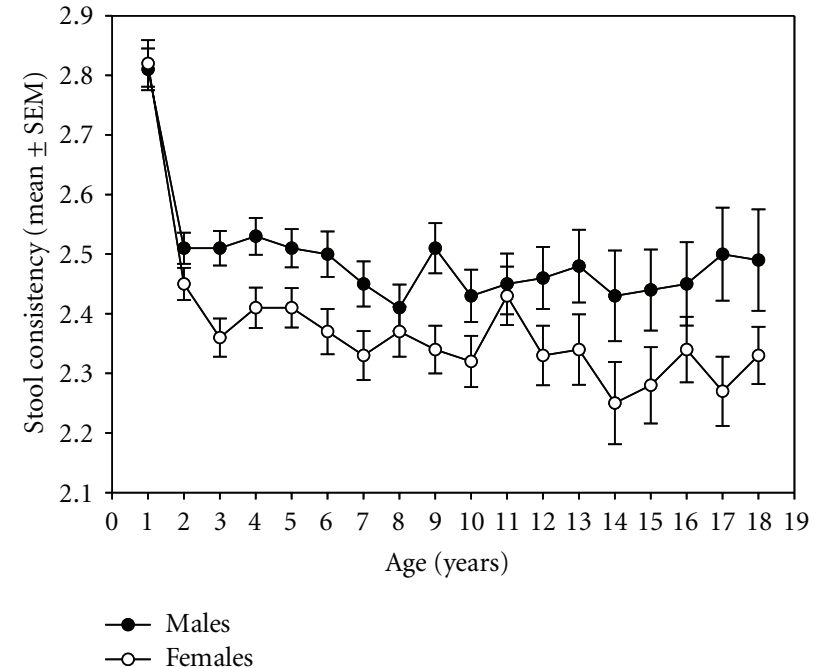

(a)

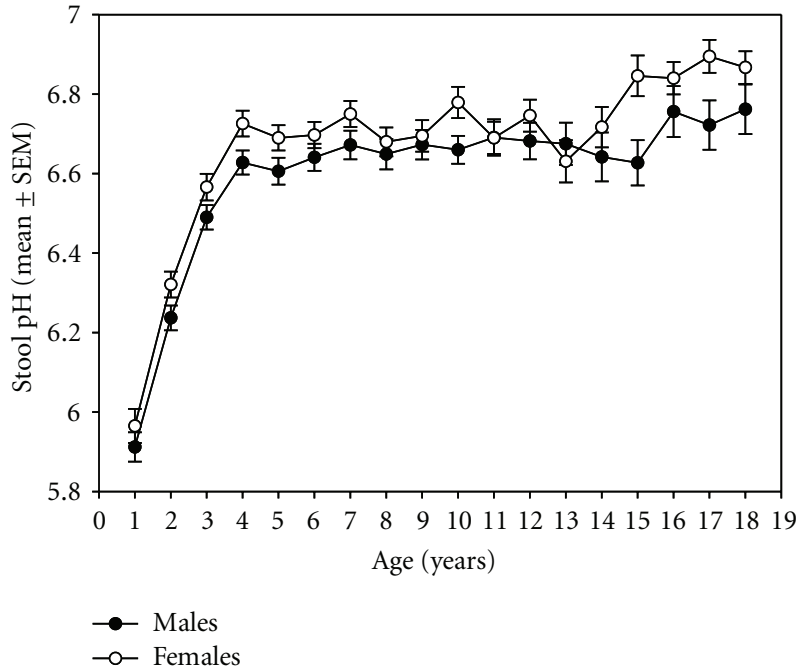

(b)

FIGURE 6: Stool consistency (mean \pm SEM) (a) ( 1 = solid, 5 = liquid) and stool pH (mean \pm SEM) (b) by gender and age (years).

Specifically, E. coli, Enterobacter sp., and Enterococci colonize the gastrointestinal tract rapidly, and are often found in higher concentrations in newborns than in adults due to the fact that aerobes and facultative anaerobes are favored by the intestinal milieu at birth. When these species expand, they consume oxygen and allow anaerobic strains to follow, including Bacteroides, Bifidobacteria, and Clostridia. Lactobacilli are oxygen tolerant and are most likely derived from the mothers' fecal flora [31]. Infants delivered by Cesarian section do not come into contact with the maternal flora during delivery. Their colonization of Bacteroides $s p$. is delayed. This has been shown to be responsible for the development of atopic dermatitis later in life [32].

However, among infants, the variability of the composition and profile of intestinal microbiota remains high [33]. It is also subject to change according to differences in nutrition [34] and other cultural influences [35], as well as host genetics, which exert a strong influence over the composition of the fecal microflora [36].

Therefore, the exploration of the "normal" range of the gut microbiota composition during the development and maturation remains a methodological challenge. In a previous paper [29], we reported the data from a large (>35.000 samples) adult cohort under the assumption that the average bacterial abundance across all patients may represent an approximation of what may be called the "normal" human fecal microbiota. Using the current data from the same source, we applied this argument to infants and children.

In agreement with previous reports (summarized by Adlerberths et al. [31]), we report a high total bacterial load in the newborn intestine within the first months after birth that declines within the first years of life to levels that are maintained during childhood and thereafter [29]. The total CFU may also reflect changes associated with weaning, since a local trough can be found (see Figure 2) at around age
7 months, which may reflect this change in nutrition. This seems somewhat counter-intuitive, as one would expect to find an increase in the CFU once more types of foods are introduced in the diet. However, weaning may induce an initial decline of predominantly lactate acid metabolizing bacteria while other species may follow colonizing the colon at a slower rate.

During the first year, the composition of the microbiota changes. E. coli and Enterococci decline, while Bacteroides sp. increases 3-folds. Bifidobacteria B. in the infant stool samples remained stable during the first years. Different from many previous reports [31], Lactobacilli were not present during the first month, but represented as much as $10 \%$ of the identified CFU one month later (Figure 3). Controversy in literature on whether or not Lactobacilli are part of the early gut colonizers has been noted [31], and it has been stated that the Lactobacilli colonizing the infant's gut are likely not from their mothers' vaginal flora [37], but rather from maternal gut flora. Colonization with Lactobacilli is delayed after Cesarian section, but reported to be normal one month later [38]. Since we do not know the route of birth in our sample, we cannot exclude that this may be the cause of the late occurrence of LB in our study. Other reasons postulated [31] for this discrepancy are differences in the methods used to identify Lactobacilli, or differences in the colonization pattern during the last decades, as many studies derive from work earlier than 1990.

During the remaining childhood up to age 10, the total CFU constantly declines to levels at about $2 \times 10$ E11 (see Figure 4), while individual strains reach stable levels at age 3 (see Figure 5). These findings also support previous reports [4, 31]. Similar to our adult sample [29], females had significantly lower stool consistency (towards more solid stools) and higher stool pH. Although both measures are not linked, they likely express the same function. We cannot exclude, however, that this may be due to differences in body 
weight and/or body mass. Unfortunately, the database did not allow adjusting for this.

Several other limitations of our analysis need to be addressed. One is that methods based on bacterial culture might not detect all species of the human microbiota. Additionally, obligate anaerobic bacteria might be at a disadvantage, and, therefore, the proportion of anaerobic bacteria to aerobic bacteria might not reflect the ratios in vivo. However, direct comparison of conventional culture analyses and molecular techniques have produced similar results, at least for the most abundant bacteria [39]. Other limitations refer to missing clinical data on medication intake, such as antibiotics. Nutritional habits and regular consumption of pre- and probiotic products may also have corroborated the results. For example, breastfeeding markedly influences the microbiotic flora in infants as does type of formula consumed and the time that supplementary food is introduced. Thus, we can only give an approximation of what may be called the 'normal' human faecal microbiota for this time period. Furthermore, the use of antibiotics severely influences intestinal flora. Since bacterial infection of the upper and lower respiratory tract is quite common, many children receive antibiotics during the first year of life. As these data were missing in our dataset, we cannot generalize our assumption. However, as stated in a previous paper [29], we reported the data from a large infant and child cohort (>12,000 samples) under the assumption that the average bacterial abundance across all patients may represent an approximation of what may be called the "normal" human fecal microbiota during infancy and childhood.

\section{Acknowledgments}

P. Enck received an unrestricted a research grant from the sponsoring company for conducting the study, and is a Consultant of the sponsoring company (SymbioPharm, Herborn, Germany). K. Zimmermann, K. Rusch, and A. Schwiertz are employees of the sponsoring company. P. Enck and J. S. Frick designed the analysis and wrote the paper, S. Klosterhalfen performed the statistical analysis, K. Zimmermann and A. Schwiertz provided the data and the details on the microbiological analysis, K. Rusch conceived the study, and participated in its design and coordination, and helped to draft the manuscript. All authors read and approved the final manuscript.

\section{References}

[1] C. D. Ramsey and J. C. Celedón, "The hygiene hypothesis and asthma," Current Opinion in Pulmonary Medicine, vol. 11, no. 1, pp. 14-20, 2005.

[2] G. Zoppi, M. Cinquetti, A. Luciano, A. Benini, A. Muner, and E. Bertazzoni Minelli, "The intestinal ecosystem in chronic functional constipation," Acta Paediatrica, vol. 87, no. 8, pp. 836-841, 1998.

[3] K. W. Mah, B. Björkstén, B. W. Lee, et al., "Distinct pattern of commensal gut microbiota in toddlers with eczema," International Archives of Allergy and Immunology, vol. 140, no. 2, pp. 157-163, 2006.
[4] J. Penders, C. Thijs, P. A. van den Brandt, et al., "Gut microbiota composition and development of atopic manifestations in infancy: the KOALA Birth Cohort Study," Gut, vol. 56, no. 5, pp. 661-667, 2007.

[5] M. Sugiyama, H. Arakawa, K. Ozawa, et al., "Early-life risk factors for occurrence of atopic dermatitis during the first year," Pediatrics, vol. 119, no. 3, pp. e716-e723, 2007.

[6] P. Bager, J. Wohlfahrt, and T. Westergaard, "Caesarean delivery and risk of atopy and allergic disesase: meta-analyses," Clinical and Experimental Allergy, vol. 38, no. 4, pp. 634-642, 2008.

[7] F. Nowrouzian, B. Hesselmar, R. Saalman, et al., "Escherichia coli in infants' intestinal microflora: colonization rate, strain turnover, and virulence gene carriage," Pediatric Research, vol. 54, no. 1, pp. 8-14, 2003.

[8] H. Renz-Polster, M. R. David, A. S. Buist, et al., "Caesarean section delivery and the risk of allergic disorders in childhood," Clinical and Experimental Allergy, vol. 35, no. 11, pp. 1466-1472, 2005.

[9] A. E. Wold, "The hygiene hypothesis revised: is the rising frequency of allergy due to changes in the intestinal flora?" Allergy, vol. 53, supplement 46, pp. 20-25, 1998.

[10] H. Garn and H. Renz, "Epidemiological and immunological evidence for the hygiene hypothesis," Immunobiology, vol. 212, no. 6, pp. 441-452, 2007.

[11] E. von Mutius, "Allergies, infections and the hygiene hypothesis-the epidemiological evidence," Immunobiology, vol. 212, no. 6, pp. 433-439, 2007.

[12] A. Sheikh and D. P. Strachan, "The hygiene theory: fact or fiction?" Current Opinion in Otolaryngology and Head and Neck Surgery, vol. 12, no. 3, pp. 232-236, 2004.

[13] D. J. Martino, H. Currie, A. Taylor, P. Conway, and S. L. Prescott, "Relationship between early intestinal colonization, mucosal immunoglobulin a production and systemic immune development," Clinical and Experimental Allergy, vol. 38, no. 1, pp. 69-78, 2008.

[14] E. Bruzzese, R. B. Canani, G. De Marco, and A. Guarino, "Microflora in inflammatory bowel diseases: a pediatric perspective," Journal of Clinical Gastroenterology, vol. 38, supplement 6, pp. S91-S93, 2004.

[15] M. J. Hudson, M. J. Hill, P. R. Elliott, et al., "The microbial flora of the rectal mucosa and faeces of patients with Crohn's disease before and during antimicrobial chemotherapy," Journal of Medical Microbiology, vol. 18, no. 3, pp. 335-345, 1984.

[16] L. V. McFarland and S. Dublin, "Meta-analysis of probiotics for the treatment of irritable bowel syndrome," World Journal of Gastroenterology, vol. 14, no. 17, pp. 2650-2661, 2008.

[17] S. Nikfar, R. Rahimi, F. Rahimi, S. Derakhshani, and M. Abdollahi, "Efficacy of probiotics in irritable bowel syndrome: a meta-analysis of randomized, controlled trials," Diseases of the Colon and Rectum, vol. 51, no. 12, pp. 1775-1780, 2008.

[18] H. Szymański, M. Armańska, K. Kowalska-Duplaga, and H. Szajewska, "Bifidobacterium longum PL03, Lactobacillus rhamnosus KL53A, and Lactobacillus plantarum PL02 in the prevention of antibiotic-associated diarrhea in children: a randomized controlled pilot trial," Digestion, vol. 78, no. 1, pp. 13-17, 2008.

[19] B. C. Johnston, A. L. Supina, M. Ospina, and S. Vohra, "Probiotics for the prevention of pediatric antibiotic-associated diarrhea," Cochrane Database of Systematic Reviews, no. 2, Article ID CD004827, 2007.

[20] S. I. Doron, P. L. Hibberd, and S. L. Gorbach, "Probiotics for prevention of antibiotic-associated diarrhea," Journal of Clinical Gastroenterology, vol. 42, supplement 2, pp. S58-S63, 2008. 
[21] S. Guandalini, "Probiotics for children with diarrhea: an update," Journal of clinical gastroenterology, vol. 42, supplement 2, pp. S53-S57, 2008.

[22] D. A. Osborn and J. K. Sinn, "Probiotics in infants for prevention of allergic disease and food hypersensitivity," Cochrane Database of Systematic Reviews, no. 4, Article ID CD006475, 2007.

[23] R. J. Boyle, F. J. Bath-Hextall, J. Leonardi-Bee, D. F. Murrell, and M. L. Tang, "Probiotics for treating eczema," Cochrane Database of Systematic Reviews, no. 4, Article ID CD006135, 2008.

[24] S. Arslanoglu, G. E. Moro, J. Schmitt, L. Tandoi, S. Rizzardi, and G. Boehm, "Early dietary intervention with a mixture of prebiotic oligosaccharides reduces the incidence of allergic manifestations and infections during the first two years of life," Journal of Nutrition, vol. 138, no. 6, pp. 1091-1095, 2008.

[25] A. Huurre, K. Laitinen, S. Rautava, M. Korkeamaki, and E. Isolauri, "Impact of maternal atopy and probiotic supplementation during pregnancy on infant sensitization: a doubleblind placebo-controlled study," Clinical and Experimental Allergy, vol. 38, no. 8, pp. 1342-1348, 2008.

[26] G. V. Zuccotti, F. Meneghin, C. Raimondi, et al., "Probiotics in clinical practice: an overview," Journal of International Medical Research, vol. 36, supplement 1, pp. 1A-53A, 2008.

[27] S. Salminen and M. Gueimonde, "Gut microbiota in infants between 6 and 24 months of age," Nestlé Nutrition Workshop Series. Paediatric Programme, vol. 56, pp. 43-51, 2005.

[28] S. Mueller, K. Saunier, C. Hanisch, et al., "Differences in fecal microbiota in different European study populations in relation to age, gender, and country: a cross-sectional study," Applied and Environmental Microbiology, vol. 72, no. 2, pp. 1027-1033, 2006.

[29] P. Enck, K. Zimmermann, K. Rusch, A. Schwiertz, S. Klosterhalfen, and J. Frick, "The effects of ageing on the colonic bacterial microflora in adults," Zeitschrift für Gastroenterologie, vol. 47, no. 7, pp. 653-658, 2009.

[30] P. M. Heavey and I. R. Rowland, "The gut microflora of the developing infant: microbiology and metabolism," Microbial Ecology in Health and Disease, vol. 11, no. 2, pp. 75-83, 1999.

[31] I. Adlerberths, L. A. Hanson, and A. E. Wold, "Ontogeny of the intestinal flora," in Development of the Gastrointestinal Tract, I. R. Sanderson and W. A. Walker, Eds., pp. 279-292, B. C. Decker, Hamilton, Canada, 1999.

[32] R. Bennet and C. E. Nord, "Development of the faecal anaerobic microflora after caesarean section and treatment with antibiotics in newborn infants," Infection, vol. 15, no. 5, pp. 332-336, 1987.

[33] C. Palmer, E. M. Bik, D. B. DiGiulio, D. A. Relman, and P. O. Brown, "Development of the human infant intestinal microbiota," PLoS Biology, vol. 5, no. 7, article e177, 2007.

[34] J. Dicksved, H. Flöistrup, A. Bergström, et al., "Molecular fingerprinting of the fecal microbiota of children raised according to different lifestyles," Applied and Environmental Microbiology, vol. 73, no. 7, pp. 2284-2289, 2007.

[35] W. M. Ka, P. Sangsupawanich, W. Tunyapanit, et al., "Gut microbiota of children living in rural south Thailand and urban Singapore," Allergology International, vol. 57, no. 1, pp. 65-71, 2008.

[36] J. A. Stewart, V. S. Chadwick, and A. Murray, "Investigations into the influence of host genetics on the predominant eubacteria in the faecal microflora of children," Journal of Medical Microbiology, vol. 54, no. 12, pp. 1239-1242, 2005.
[37] G. W. Tannock, R. Fuller, S. L. Smith, and M. A. Hall, "Plasmid profiling of members of the family Enterobacteriaceae, lactobacilli, and bifidobacteria to study the transmission of bacteria from mother to infant," Journal of Clinical Microbiology, vol. 28, no. 6, pp. 1225-1228, 1990.

[38] M. A. Hall, C. B. Cole, S. L. Smith, R. Fuller, and C. J. Rolles, "Factors influencing the presence of faecal lactobacilli in early infancy," Archives of Disease in Childhood, vol. 65, no. 2, pp. 185-188, 1990.

[39] C. Liu, Y. Song, M. McTeague, A. W. Vu, H. Wexler, and S. M. Finegold, "Rapid identification of the species of the Bacteroides fragilis group by multiplex PCR assays using group- and species-specific primers," FEMS Microbiology Letters, vol. 222, no. 1, pp. 9-16, 2003. 


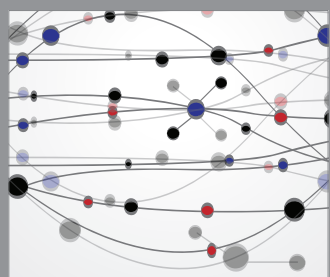

The Scientific World Journal
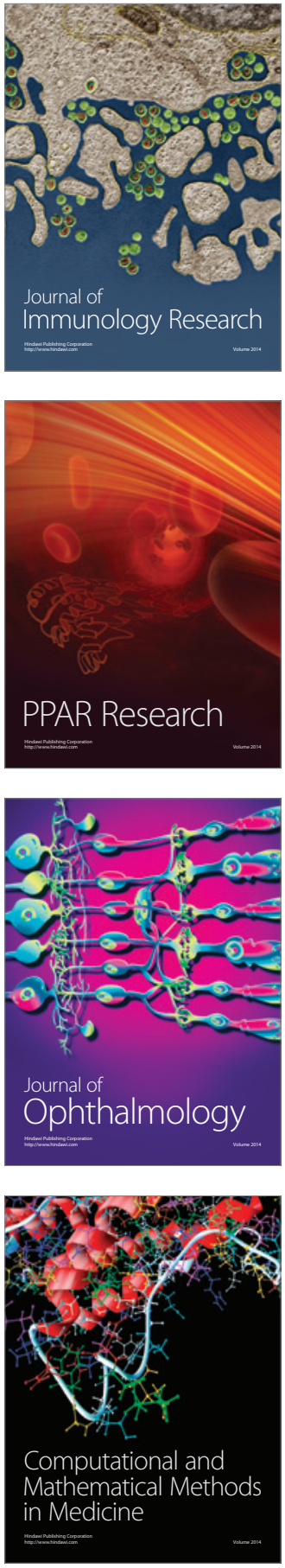

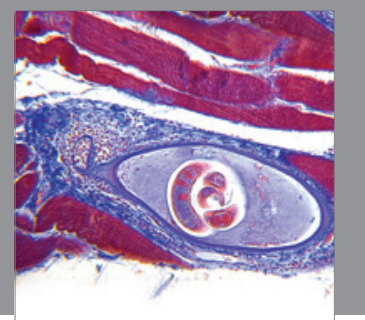

Gastroenterology

Research and Practice
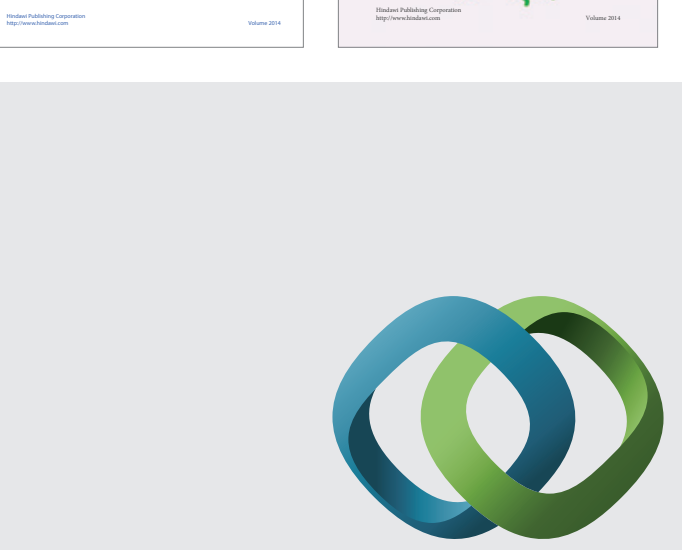

\section{Hindawi}

Submit your manuscripts at

http://www.hindawi.com
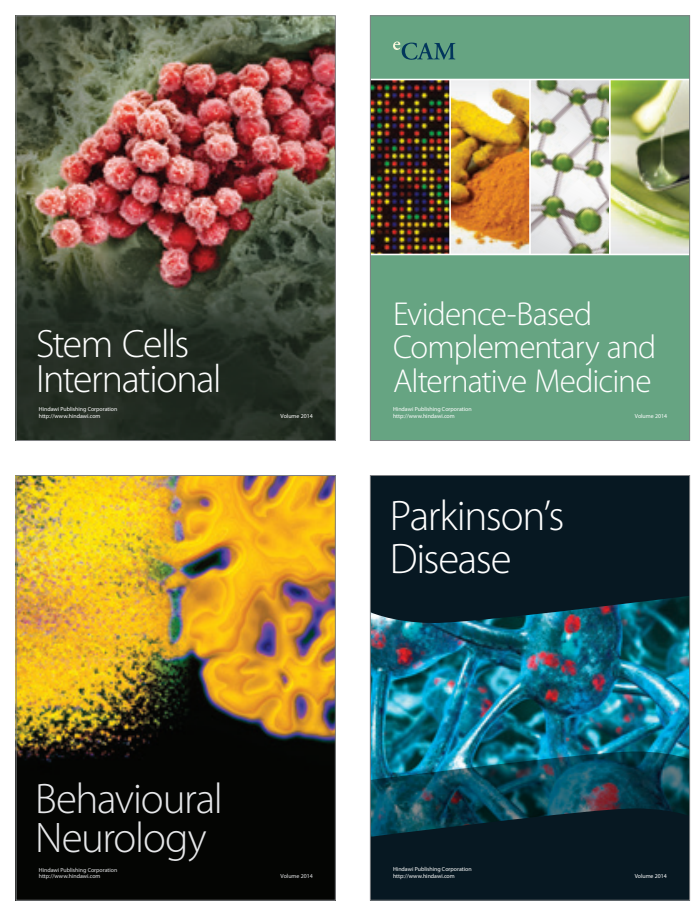

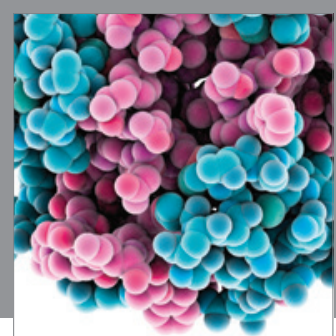

Journal of
Diabetes Research

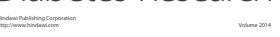

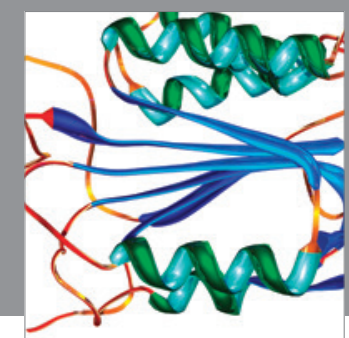

Disease Markers
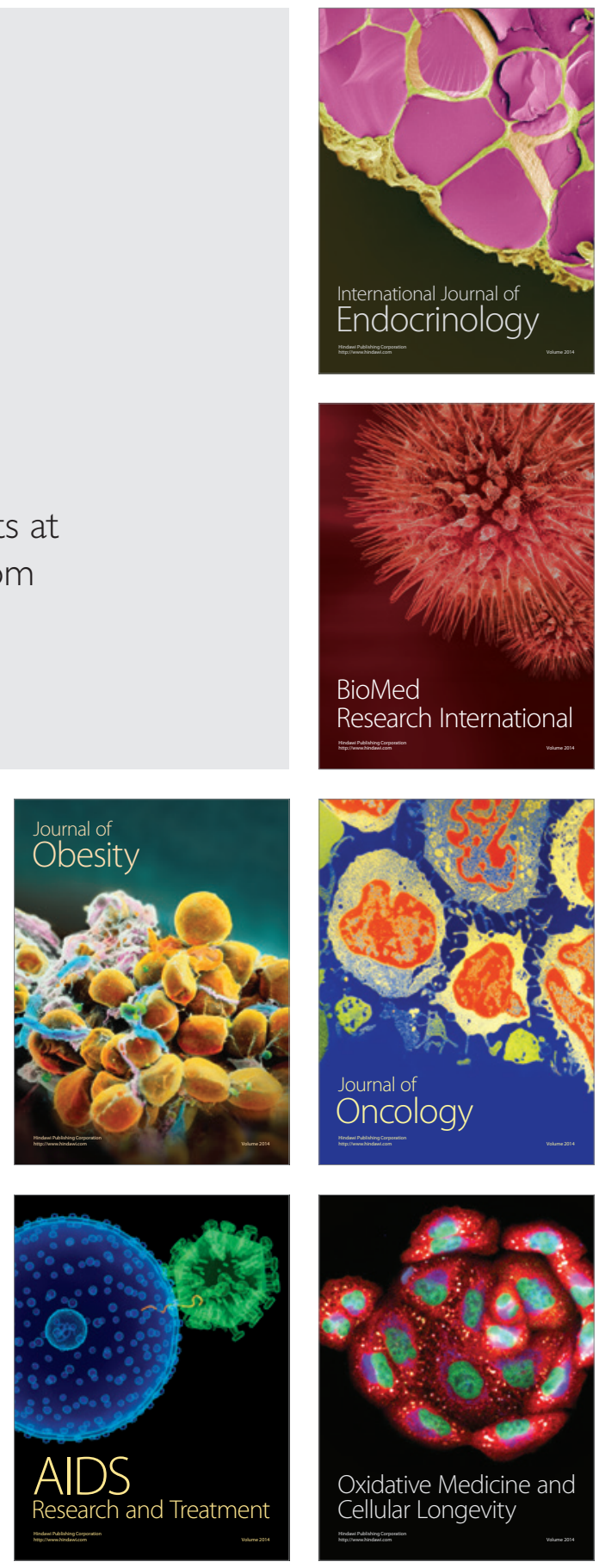